\title{
Fluorescence Lifetime Correlation Spectroscopy Combined with Lifetime Tuning: New Perspectives in Supported Phospholipid Bilayer Research
}

\author{
Aleš Benda, ${ }^{\dagger}$ Veronika Fagul’ová, ${ }^{\dagger}$ Alexander Deyneka, ${ }^{\ddagger}$ Joerg Enderlein, ${ }^{\S}$ and \\ Martin Hof*,† \\ J. Heyrovský Institute of Physical Chemistry, Academy of Sciences of the Czech Republic, Dolejškova 3, \\ 18223 Praha 8, Czech Republic, Institute of Physics, Academy of Sciences of the Czech Republic, \\ Na Slovance 2, 18221 Praha 8, Czech Republic, and Institute for Biological Information Processing 1, \\ Forschungszentrum Jülich, D-52425 Jülich, Germany
}

Received June 1, 2006. In Final Form: August 25, 2006

\begin{abstract}
A new concept based on fluorescence lifetime correlation spectroscopy (FLCS) is presented allowing the simultaneous determination of diffusion coefficients of identical molecules located in different environments. The difference in fluorescence lifetimes, which is the main prerequisite for FLCS, is reached by locating one population of the dye close to a light-absorbing surface. Since such surfaces quench fluorescence, the fluorescence lifetime of chromophores located close to these surfaces can be tuned in a specific manner. This approach has been demonstrated for a BODIPYtail-labeled lipid in supported phospholipid bilayers (SPBs) as well as in phospholipid multilayers adsorbed onto solid supports. In particular, the effect of the solid support type on the fluorescence lifetime as well as its dependence on the BODIPY-support distance has been characterized and verified by theoretical considerations based on precise determination of refractive indices of the used supports. While the fluorescence lifetime of BODIPY dye is 5.6 ns in small unilamellar vesicles (SUVs) composed of 1,2-dioleoyl-sn-glycero-3-phosphocholine (DOPC) and 1,2-dioleoylsn-glycero-3-[phospho-L-serine] (DOPS), the lifetime is $1.8 \mathrm{~ns}$ in DOPC/DOPS SPBs adsorbed onto ITO-covered glass or $3.0 \mathrm{~ns}$ in a DOPC/DOPS monolayer adsorbed onto seven 1,2-dipalmitoyl-sn-glycero-3-phosphate (DPPA) layers on oxidized silicon. Using these particular systems, we demonstrated that FLCS enables one to characterize simultaneously two-dimensional lipid diffusion in the planar lipid layers and three-dimensional vesicle diffusion in bulk above the lipid layers using single dye labeling. The autocorrelation functions obtained by this new approach do agree with those obtained by standard FCS on isolated SPBs or vesicles. Possible applications of this virtual two-channel measurement using single dye labeling as well as one detection channel are discussed.
\end{abstract}

\section{Introduction}

Fluorescence correlation spectroscopy (FCS) has been used recently to determine lateral diffusion coefficients of labeled lipids or lipid analogues within cells ${ }^{1,2}$ giant unilamellar vesicles, ${ }^{3}$ and supported phospholipids bilayers (SPBs). ${ }^{2,4,5}$ In particular, our group worked out a protocol for determination of diffusion coefficients and surface densities in planar phospholipid systems ${ }^{4}$ and showed that the so-called "Z-scan" approach is an indispensable tool for reproducible and precise determination of these parameters. ${ }^{2}$ Recently, the measurement capabilities of FCS have been enriched using pulsed lasers as well as time-tagged timeresolved (TTTR) data storage. Similarly to two-color FCS, ${ }^{6}$ in such a fluorescence lifetime correlation spectroscopy ${ }^{7,8}$ (FLCS)

* Corresponding author: Contact e-mail address: hof@jh-inst.cas.cz.

$\dagger$ J. Heyrovský Institute of Physical Chemistry, Academy of Sciences of the Czech Republic.

$\stackrel{+}{\dagger}$ Institute of Physics, Academy of Sciences of the Czech Republic.

$\S$ Institute for Biological Information Processing 1.

(1) Schwille, P.; Haupts, U.; Maiti, S.; Webb, W. W. Biophys. J. 1999, 77 (4), 2251-2265.

(2) Humpolickova, J.; Gielen, E.; Benda, A.; Fagulova, V.; Vercammen, J.; Vandeven, M.; Hof, M.; Ameloot, M.; Engelborghs, Y. Biophys. J. 2006, 91 (3), L23-L25.

(3) Kahya, N.; Scherfeld, D.; Bacia, K.; Schwille, P. J. Struct. Biol. 2004, 147 (1), 77-89.

(4) Benda, A.; Benes, M.; Marecek, V.; Lhotsky, A.; Hermens, W. T.; Hof, M. Langmuir 2003, 19 (10), 4120-4126.

(5) Zhang, L. F.; Granick, S. J. Chem. Phys. 2005, 123 (21), Art. No. 211104. (6) Schwille, P.; MeyerAlmes, F. J.; Rigler, R. Biophys. J. 1997, 72 (4), $1878-$ 1886.

(7) Böhmer, M.; Wahl, M.; Rahn, H. J.; Erdmann, R.; Enderlein, J. Chem. Phys. Lett. 2002, 353 (5-6), 439-445.

(8) Enderlein, J.; Gregor, I. Rev. Sci. Instrum. 2005, 76 (3), Art. No. 033102. experiment labeling two different biomolecules or bioaggregates with two different chromophores differing in their fluorescence lifetimes $(\tau(\mathrm{F}))$ enables one to record simultaneously the autocorrelation functions of individual labeled species as well as compute the corresponding cross-correlation functions. ${ }^{9}$ While in two-color FCS the chromophores have to exhibit large differences in their spectral characteristics, sound FLCS experiments can be performed in a straightforward manner if the fluorescence lifetimes of the two chromophores with identical or similar spectral characteristics differ by a factor of about 2 or more. Since the fluorescence lifetime of a chromophore is sensitive to changes in its particular microenvironment or to intentional addition of quenchers, FLCS offers a unique advantage: two different diffusion processes can be characterized using single dye labeling. It should be pointed out that one can hardly imagine conditions which would lead to environmentdriven spectral shifts which are sufficiently large for using twocolor FCS. In this work we exploit changes in the fluorescence lifetime of a labeled lipid in SPBs in the presence of an interface (solid support). Light-absorbing supports quench the lifetime of a dye located within nanometer's distance to the support to a value significantly shorter than the lifetime of the same dye located in freely diffusing small unilamellar vesicles (SUVs). To this end we first characterized how the lifetime of labeled lipids in SPBs can be tuned by varying the solid support as well as the dye-support distance. Second, we have shown that after preparing SPBs from labeled vesicles, FLCS enables one to characterize

(9) Benda, A.; Hof, M.; Wahl, M.; Patting, M.; Erdmann, R.; Kapusta, P. Rev. Sci. Instrum. 2005, 76 (3), Art. No. 033106. 


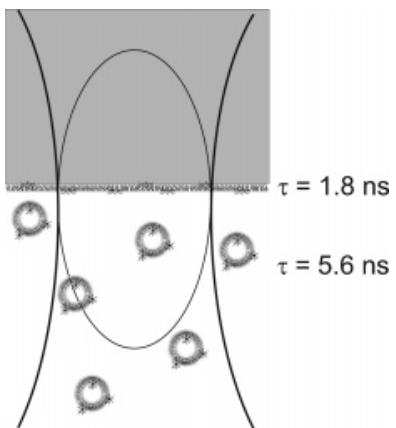

Figure 1. Illustration of an FLCS experiment for simultaneous characterization of two- and three-dimensional diffusion. The main idea is that the excited-state lifetime of the fluorophore in SPBs (for example, $1.8 \mathrm{~ns}$ for BODIPY tail-labeled lipid in SPBs on glass covered with $173 \mathrm{~nm}$ ITO layer) is shorter than the excited-state lifetime of the same dye in solution (5.6 ns for BODIPY tail-labeled lipid in SUVs). This difference in lifetimes enables one to apply FLCS for separating autocorrelation curves of these two populations of the same dye. The diameter of SUVs is $50 \pm 10 \mathrm{~nm}$, the beam waist radius is around $250 \mathrm{~nm}$, and the bilayers assumed to be $6 \mathrm{~nm}$.

simultaneously two-dimensional lipid diffusion in SPBs and threedimensional vesicle diffusion in a single experiment (Figure 1). Autocorrelation curves obtained by this new approach have been compared to those obtained by standard FCS on isolated SPBs or vesicles.

\section{Material and Methods}

Chemicals and Supports Used and Their Cleaning. Lipids 1,2-dioleoyl-sn-glycero-3-phosphocholine (DOPC), 1,2-dioleoylsn-glycero-3-[phospho-1-serine] (sodium salt) (DOPS), and 1,2dipalmitoyl-sn-glycero-3-phosphate (monosodium salt) (DPPA) were purchased from Avanti Polar Lipids, Inc. (Alabaster, AL). 2(4,4-Difluoro-5-octyl-4-bora-3a,4a-diaza-s-indacene-3-pentanoyl)-1-hexadecanoyl-sn-glycero-3-phosphocholine ( $\beta$-C8-BODIPY 500/510 C5-HPC) was purchased from Molecular Probes, Inc. (Willow Creek Road, Eugene, OR). All chemicals needed for buffer preparation were purchased from Sigma-Aldrich. Water used in our study was purified with the MilliQ purification system. Buffer A for SUVs preparation contained $0.1 \mathrm{mM}$ EDTA, $10 \mathrm{mM}$ Hepes, and $150 \mathrm{mM} \mathrm{NaCl}(\mathrm{pH}$ 7.4). Buffer B for SPBs incubation and measurement contained $2 \mathrm{mM} \mathrm{CaCl}_{2}, 10 \mathrm{mM}$ Hepes, and $150 \mathrm{mM}$ $\mathrm{NaCl}(\mathrm{pH}$ 7.4). The buffers were filtered through a $0.2 \mu \mathrm{m}$ syringe filter (Schleicher \& Schuell, Germany) prior to use. Glass Coverslipe (Marienfeld, Germany), phosphor-doped silicon wafer (WaferNet, Inc., CA), quartz slide (Ted Pella, Inc., Redding, CA), and ITO (glass covered with a 173-nm layer of indium-tin oxide (90\% indium oxide $\mathrm{In}_{2} \mathrm{O}_{3}$ and $10 \%$ tin oxide $\mathrm{SnO}_{2}$; GeSiM Grosserkmannsdorf Germany) were cleaned before use with a detergent (Sparkleen, Fisher Scientifc Co., Pittsburgh, PA), rinsed with MilliQ water, dried under a flow of nitrogen, and subjected for $10 \mathrm{~min}$ to a UV/ozone treatment (a homemade chamber with a mercury grid lamp (B.H.K., Inc., CA)). Mica (muscovite) (JBG-METAFIX, Montdidier, France) was cleaved before use using scotch tape.

Characterization of Optical Properties of Individual Surfaces. Refractive indices of individual surfaces and layer thicknesses were measured with a J. A. Woollam spectral ellipsometer operating in the rotating analyzer mode. The main ellipsometric angles $\Psi$ and $\Delta$ were measured in the spectral range from 300 to $1000 \mathrm{~nm}$ at three angles of incidence in air. The ellipsometric data were analyzed using the software package WVASE32. A simple substrate/film/ roughness model was used to calculate the optical constants.

Preparation of SPBs and Cuvettes for FCS Measurements. First, a mixture of labeled and unlabeled lipids was prepared by mixing appropriate amounts of these lipids dissolved in chloroform. The solvent was evaporated by a stream of dry nitrogen, and the lipid mixture was resuspended by vortexing in an appropriate volume of buffer A to form a turbid suspension. The latter was sonicated

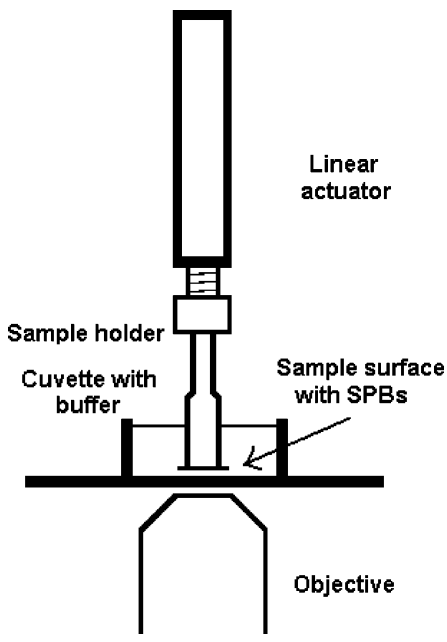

Figure 2. Schematic representation of the setup used in FCS measurement on supported phospholipid bilayers deposited on different supports. The support with SPBs is placed on the hollow glass rod attached to a linear actuator. The support is brought to a working distance of the objective $(200 \mu \mathrm{m})$ and aligned perpendicularly to optical axis.

by a tip sonicator (SONOPULS HD 2070, BANDELIN electronic GmbH \& Co. KG, Berlin, Germany) for 20 min to yield a solution of small unilamellar vesicles (SUVs). The ratio of labeled to unlabeled lipid was $1: 10^{5}$.

The experimental configuration for FCS measurements on SPBs is presented in Figure 2. The supports were fixed to hollow glass rods (5 $\mathrm{mm}$ in diameter) and mounted on a computer-controlled linear actuator with $50 \mathrm{~nm}$ minimum step (type M-230.10, PI, Karlsruhe, Germany). The sample was brought $200 \mu \mathrm{m}$ above the bottom of the cuvette (the maximum working distance of the objective) and adjusted to be perpendicular to the optical axis. The cuvette and objective were kept at $25^{\circ} \mathrm{C}$ by Peltier cells (temperature range $0-50{ }^{\circ} \mathrm{C}$, Con Brio, Pardubice, Czech Republic). The setup employed also included a flushing system and an immersion stirrer.

The cuvette with the sample (Figure 2) was filled with buffer B. A solution of SUVs (dilution 1:9 to final lipid concentration 100 $\mu \mathrm{M}$ ) was added and incubated for 30 min under continuous stirring. The quality of the SPBs was checked (a) by ellipsometry measurements ${ }^{10}$ (DRE ellipsometer, Germany) and (b) in situ by intensity scans and FCS lateral mobility measurement. ${ }^{4}$ The redundant vesicles were then flushed with buffer B.

Preparation of Langmuir-Blodgett Films. The LangmuirBlodgett films were prepared using a Nima Langmuir Blodgett trough (model 612D-RB, Nima Technology, Coventry, England). The hydrophilized samples were immersed into the subphase $(0.1 \mathrm{mM}$ $\left.\mathrm{CaCl}_{2}\right) ; 1 \mathrm{mM}$ solution of DPPA in chloroform was spread on the subphase-air interface and compressed to a surface pressure drop of $35 \mathrm{mN} / \mathrm{m}$. Odd numbers of DPPA monolayers were deposited by pulling out and dipping in at a constant pressure at a speed of 5 $\mathrm{mm} / \mathrm{min}$. Before the last dip the DPPA was exchanged for a mixture of DOPC/DOPS 4/1 labeled with $\beta$-C8-BODIPY 500/510 C5-HPC and compressed to a surface pressure drop of $40 \mathrm{mN} / \mathrm{m}$. After completion of the above procedure (even number of monolayers, only the last labeled) the sample was transferred under liquid to the measurement cuvette. The quality of multilayers was checked by transfer ratios (close to 1), and their thickness was measured on the ellipsometer. ${ }^{10}$

Time-Resolved FCS Instrument. FCS measurements were performed using an upgraded Confocor $1^{9}$ (Carl Zeiss GmbH, Jena; Evotec Biosystems GmbH, Hamburg; PicoQuant GmbH, Berlin, Germany). Briefly, it consists of an inverted confocal microscope Axiovert 100 with a water immersion objective C-Apochromat $40 \times / 1.2 \mathrm{~W}$, pulsed diode lasers LDH-P-C-470 and LDH-P-635 (470

(10) Benes, M.; Billy, D.; Benda, A.; Speijer, H.; Hof, M.; Hermens, W. T Langmuir 2004, 20 (23), 10129-10137. 
and $635 \mathrm{~nm}$, fwhm of the pulse duration is $80 \mathrm{ps)} \mathrm{with} \mathrm{PDL800-B}$ driver (PicoQuant $\mathrm{GmbH}$, Berlin, Germany), the appropriate fluorescence filter sets (Chroma Technology, Rockingham, VT), a single photon counting detector SPCM-AQR-13-FC (Perkin-Elmer, Fremont, MA), and a time-tagged time-resolved (TTTR) data storing card TimeHarp 200 (PicoQuant GmbH, Berlin, Germany). The full width at half-maximum (fwhm) of the overall instrument response function (IRF) is $550 \mathrm{ps}$ for the $470 \mathrm{~nm}$ diode laser and $460 \mathrm{ps}$ for the $635 \mathrm{~nm}$ diode laser. FCS data fitting was performed with the OriginPro70 software package (OriginLab Corp., Northampton, MA).

Data Acquisition and Analysis. All experiments were carried out using the TTTR data-storing mode. The $Z$-scan approach was applied ${ }^{4}$ when recording the TTTR datasets. Scanning along the optical axis was performed by moving the sample. The linear actuator was programmed to move in steps of $182 \mathrm{~nm}$ and after each step to send the TTL signal to the TimeHarp 200 board, which registered it and put it into the TTTR file as a marker. Lifetime fitting was performed using MicroTime 200 and FluoFit Pro software packages (PicoQuant $\mathrm{GmbH}$, Berlin, Germany). Tail fitting (starting at the first channel where IRF intensity is identical to the background) using either the mono- or biexponential model was applied.

The routines for time-resolved $\mathrm{FCS}^{7}$ data treatment and analysis were implemented in OriginPro70. The key part of the analysis, a correlation of the filtered data, was written in $\mathrm{C}(\mathrm{Dev}-\mathrm{C}++$, Bloodshed software) as a dll library. Fitting of the resulting correlation curves was performed by OriginPro70 routines (LevenbergMarquardt nonlinear least-squares routine) using the Wohland ${ }^{4,11}$ method for experimental determination of standard deviations. For lateral diffusion within the membranes a standard model for twodimensional diffusion with intersystem crossing was used

$$
G(\tau)_{2 \mathrm{DT}}=1+\left(1-T+T e^{-\tau / \tau \mathrm{tr}}\right)\left(\frac{1}{P N[1-T]}\right)\left(\frac{1}{1+\tau / \tau_{\mathrm{D}}}\right)
$$

where $P N$ and $\tau_{\mathrm{D}}$ represent the particle number and diffusion time, respectively, $T$ is the fraction of dye molecules in the triplet state, and $\tau_{\text {tr }}$ is the intersystem crossing relaxation time.

The principles of fluorescence lifetime correlation spectroscopy (FLCS) are explained in papers by Böhmer ${ }^{7}$ and Enderlein. ${ }^{8}$ In short, FLCS enables one to separate correlation functions of different lifetime contributions in a mixture. These contributions are called patterns and can originate from different fluorescence species, one species located in several different environments and/or from parasitic signals. To be able to construct the statistical filters, which are sets of coefficients separating the intensity trace into its components, the fluorescence patterns of all contributing components must be known in advance. Two basic approaches can be used. The first is to measure the patterns separately; a procedure free of any fitting that however requires that the fluorescence decays of the contributing components can be measured separately. The second is to fit the measured histogram of the mixture to a relevant decay model by an iterative convolution technique using the instrument response function (IRF). The individual patterns are then reconstructed from the corresponding fit parameters of the individual components. These two basic approaches can be combined also: one measures the lifetime histogram of one of the components separately and uses this histogram as a fixed parameter in fitting the data from the mixture. Since the fluorescence decay patterns of dye in SUVs and SPBs can be determined independently, we used the first approach, which does not involve any lifetime fitting.

\section{Results and Discussion}

Lifetime Tuning: General Aspects. The influence of a partially reflecting mirror on the fluorescence lifetime of an emitting molecule located close to it was experimentally and theoretically studied in the 1970s. ${ }^{12-14}$ The first studies focused

(11) Wohland, T.; Rigler, R.; Vogel, H. Biophys. J. 2001, 80 (6), 2987-2999. (12) Chance, R. R.; Miller, A. H.; Prock, A.; Silbey, R. J. Chem. Phys. 1975, 63 (4), 1589-1595. on the change of the fluorescence lifetime; more recent studies were mainly interested in the overall detectable fluorescence intensity from a single molecule located in stratified media. ${ }^{15-20}$ Theoretical investigations treat the system from a classical electrodynamics point of view. The fluorescent molecule is assumed to be a pointlike oscillating dipole. The radiative emission of such a dipole is influenced by its own emission electromagnetic field that is back-reflected from nearby optical interfaces. Parameters that completely describe the system are the wavelength of the emitted light, the complex refractive indices of all materials, the thicknesses of the layers, location of the molecule within the layered system, and emission dipole orientation with respect to the layers. Let us define the relative damping constant $k_{\mathrm{rel}}=k_{\mathrm{s}} / k_{\mathrm{r}}$ as the ratio of the total energy flux from the molecule in the system of interest $k_{\mathrm{s}}$ and in the reference system $k_{\mathrm{r}}$ and the relative lifetime $\tau_{\text {rel }}=\tau_{\mathrm{s}} / \tau_{\mathrm{r}}$ as the ratio of the measured lifetime in the system of interest $\tau_{\mathrm{s}}$ and the reference system $\tau_{\mathrm{r}}$. The relation between the relative lifetime and the relative damping constant is then $1 / \tau_{\text {rel }}=Q_{\mathrm{r}}\left(k_{\mathrm{rel}}-1\right)+1$, where $Q_{\mathrm{r}}$ is the quantum yield of the dye in the reference system. We assume that the rates of other nonradiative deactivation processes are the same for both the sample and the reference systems.

There are two main mechanisms underlying a change in the lifetime. The first is the rather moderate effect of optical interference leading to an oscillatory behavior at long distances (on the order of the emission wavelength). The second is a strong electromagnetic coupling between the fluorescing molecule and the absorbing surface (a metal or a semiconductor with an imaginary part of the refractive index) leading to a strong energy transfer from the molecule to the surface and resulting in a shortrange (tens of nanometers) quenching and lifetime decrease.

Lifetime Tuning: Influence of the Support. As already mentioned, the fluorescent lifetime of a molecule close to an optical interface is strongly influenced by the optical properties of the interface. SPBs created by vesicle fusion can be generally prepared on many surfaces. ${ }^{10,21} \mathrm{We}$ chose five of them (glass, mica, silica, ITO, oxidized silicon) to assess the effect of different refractive indices of the support on the fluorescent lifetime of a dye molecule embedded in SPBs.

Supports were exposed to SUV suspensions containing taillabeled lipid $\beta$ - $\mathrm{C}_{8}$-BODIPY 500/510 $\mathrm{C}_{5}$-HPC. The BODIPY dyes have a quantum yield of around $0.93 .^{22}$ After incubation, measurements on SUVs in buffer (far away from any surface) were performed in order to obtain a reference for lifetime values. Redundant vesicles were then flushed away, and TTTR Z-scan FCS measurements on the SPBs were performed. Lateral mobility was checked by evaluating the autocorrelation functions to assess the quality of the SPBs. ${ }^{4}$ In all cases, confluent bilayers were found (data not shown). Lifetime histograms were constructed from the TTTR data and fitted. The obtained lifetime values are summarized in Table 1. One lifetime was sufficient for all histograms to fit the fluorescence decay by means of tail fitting.

(13) Chance, R. R.; Prock, A.; Silbey, R. J. Chem. Phys. 1974, 60 (7), 27442748.

(14) Chance, R. R.; Prock, A.; Silbey, R. J. Chem. Phys. 1975, 62 (6), 22452253.

(15) Enderlein, J. Chem. Phys. 1999, 247 (1), 1-9.

(16) Vasilev, K.; Knoll, W.; Kreiter, M. J. Chem. Phys. 2004, 120 (7), 34393445 .

(17) Kreiter, M.; Prummer, M.; Hecht, B.; Wild, U. P. J. Chem. Phys. 2002, 117 (20), 9430-9433.

(18) Vasilev, K.; Stefani, F. D.; Jacobsen, V.; Knoll, W.; Kreiter, M. J. Chem. Phys. 2004, 120 (14), 6701-6704.

(19) Enderlein, J. Biophys. J. 2000, 78 (4), 2151-2158.

(20) Enderlein, J.; Böhmer, M. Opt. Lett. 2003, 28 (11), 941-943.

(21) Tamm, L. K.; Mcconnell, H. M. Biophys. J. 1985, 47 (1), 105-113.

(22) Karolin, J.; Johansson, L. B. A.; Strandberg, L.; Ny, T. J. Am. Chem. Soc. 1994, 116 (17), 7801-7806. 
Table 1. Refractive Indices and Lifetimes of the Labeled Lipid $\beta$-C8-BODIPY 500/510 C5-HPC in SPBs on Different Surfaces and in SUVs in Solution ${ }^{a}$

\begin{tabular}{llcc}
\hline $\begin{array}{c}\beta \text {-C8-BODIPY } \\
\text { 500/510 C5-HPC in }\end{array}$ & $\begin{array}{c}\text { refractive indices } \\
(520 \mathrm{~nm})\end{array}$ & $\begin{array}{c}\text { lifetime } \\
(\mathrm{ns})\end{array}$ & $\begin{array}{c}\text { relative } \\
\text { lifetime }\end{array}$ \\
\hline free SUVs & 1.337 & 5.6 & 1 \\
SPBs on silica & 1.461 & 4.9 & 0.88 \\
SPBs on glass & 1.533 & 4.7 & 0.84 \\
SPBs on mica & 1.632 & 4.4 & 0.79 \\
SPBs on glass with & $1.529 ; 2.043-0.037 \mathrm{i}$ & 1.8 & 0.32 \\
$\quad$ 173 nm ITO & & & \\
SPBs on oxidized silicon & $4.193-0.020 \mathrm{i} ; 1.461$ & 1.0 & 0.18
\end{tabular}

$\left(\sim 3 \mathrm{~nm} \mathrm{SiO}_{2}\right)$

${ }^{a}$ The relative lifetime is related to the lifetime of the BODIPY dye in SUVs in solution and is also used in Figure 3.

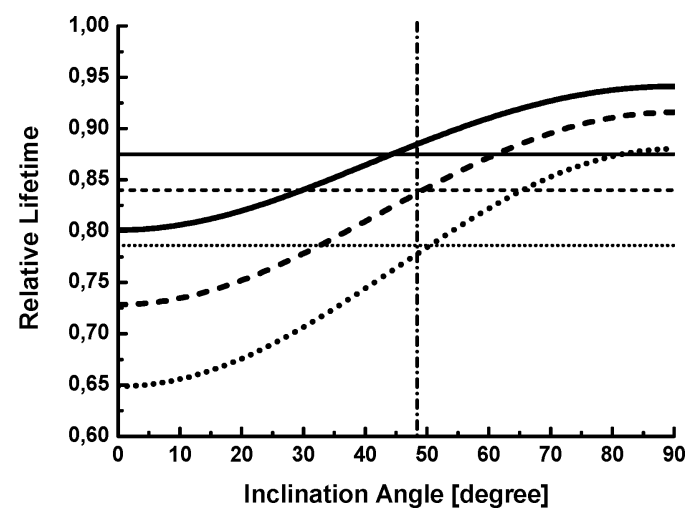

Figure 3. Calculated dependence of the relative lifetime of BODIPY dye in the middle of SPBs on (a) silica (thick solid line), (b) glass (thick dashed line), and (c) mica (thick dotted line) on their orientation. The thickness of SPBs is assumed to be $6 \mathrm{~nm}$ and the refractive index 1.45. The best match between experiment (thin lines, for relative lifetime values see Table 1) and theory is reached for the inclination angle of $48^{\circ}$.

Next, we compared the experimental values with theoretical calculations. For this comparison the relative lifetimes are needed, i.e., the measured lifetimes at the interface normalized to lifetimes in a homogeneous environment. A solution of freely diffusing SUVs (of the same composition as the SPBs) measured far away from any surface was used as the experimental reference, while a plane phospholipid bilayer surrounded on both sides by buffer was used as the theoretical reference. The values used for the calculations were emission wavelength $=520 \mathrm{~nm}$, refractive index of water (buffer) $=1.337$, refractive index of the hydrated bilayer $=1.45$, thickness of the bilayer $=6 \mathrm{~nm}$, and position of the chromophore in the center of the hydrophobic part of the bilayer. The used values for thickness and refractive index of SPBs were experimentally determined by ellipsometry. ${ }^{10}$ The refractive indices of the supports are summarized in Table 1. The last parameter is the orientation of the dye emission dipole with respect to the bilayer's normal. As this value has not been measured experimentally, we performed calculations for both principal (parallel and perpendicular) orientations. The final value of the relative lifetime is then obtained as an orientation-weighted average of both lifetimes. The calculations for dielectric supports silica, glass, and mica show (Figure 3 ) good agreement within experimental error with the experiments and result in $48^{\circ}$ as the average inclination angle of the BODIPY emission dipole in SPBs. For a dipole randomly oriented in space a value of magic angle $\left(54.7^{\circ}\right)$ would be obtained. We are aware that the calculation of these angles is dependent on many factors, like the angular distribution of the dye emission dipole, refractive indices, or emission wavelength used in the calculations. Moreover, the

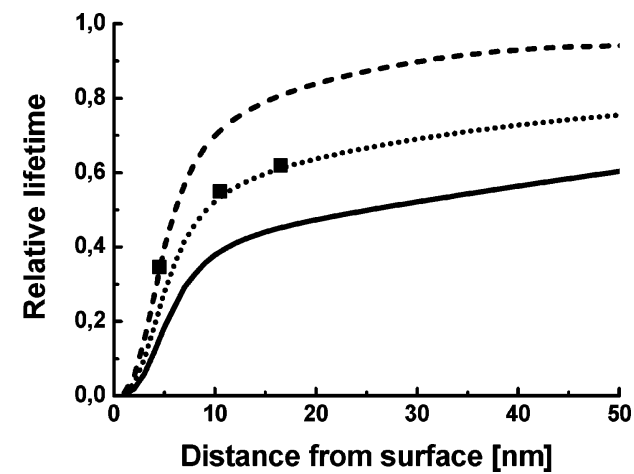

Figure 4. Theoretical and experimental dependences of the relative lifetime of BODIPY dye in lipid monolayer on its distance from the surface of an ITO layer on glass, $173 \mathrm{~nm}$ thick. The shown distance is measured relative to the surface of the support, and it is assumed that the chromophore is separated from the support by lipid layers. Data for vertical (solid line), parallel (dashed line), and space-averaged $\left(48^{\circ}\right)$ (dotted line) orientation are shown together with the experimental points (squares).

theory underlying these calculations contains several assumptions. However, for three different surfaces we obtained the same result, and we are thus confident that the value of $48^{\circ}$ reflects the real orientation.

Both the experiments and theory show that the relative lifetime shortening in SPBs on dielectric substrates (silica, glass, mica) is rather moderate and not very suitable for applications in FLCS experiments. On the contrary, light-absorbing surfaces such as ITO and silicon show much better quenching efficiency and thus seem to be suitable for further experiments.

Lifetime Tuning: Distance Dependence. Another aspect of the surface quenching which has to be considered is how the quenching efficiency propagates toward the solution. In other words, we have to know to what extent the fluorescence of the molecules in solution is quenched and thus may invalidate the concept suggested in this paper. For this reason a second type of experiment was done to characterize the dependence of lifetime quenching on the dye-surface distance for ITO and silicon surfaces. Using the Langmuir-Blodgett stacking technique odd numbers of DPPA monolayers in the gel phase were used as variable spacers between the support and the "last" BODIPYtail-labeled fluid DOPC/DOPS monolayer. The thickness of one DPPA monolayer is approximately $3 \mathrm{~nm}$. The measured relative lifetimes are compared in Figures 4 and 5 with those obtained from theoretical calculations, showing again good agreement. Actually, only the first point for ITO sample is slightly shifted to lower quenching efficiencies, which might be attributed to uncertainty in either distance determination or composition of the outmost ITO layer.

A significant difference between these two supports concerns the steepness of the dependence of the fluorescence lifetime on the dye-support distance. For ITO the lifetime shortening disappears much faster, which is convenient for the FLCS experiments. Since the final aim of this study is to use FLCS to simultaneously determine the 2D lipid diffusion in SPBs and the 3D diffusion of vesicles in solution, one has to know the distance dependence for the case where the space between the dye and the surface is not filled by DPPA multilayers with refractive index of 1.45 but only by one SPBs and a water solution with refractive index of 1.337 . Since the latter system cannot be characterized experimentally, we performed theoretical calculations; the results are presented in Figure 6.

Figures 4 and 5 show that using a defined spacer enables one to tune the lifetime in a defined way, a main prerequisite for a 


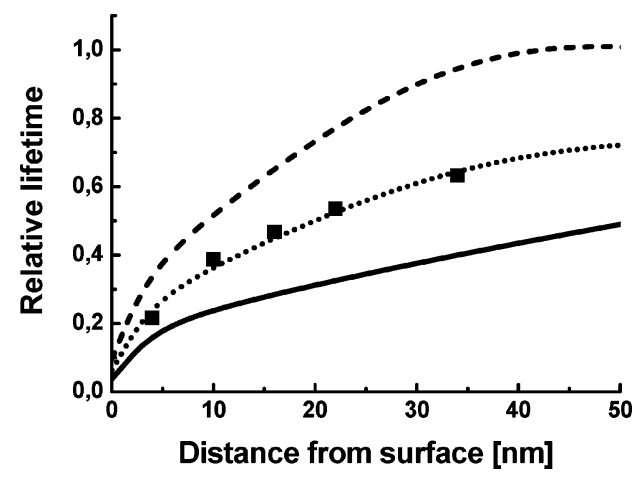

Figure 5. Theoretical and experimental dependences of the relative lifetime of BODIPY dye in lipid monolayer on its distance from the surface of naturally oxidized silicon. The shown distance is measured relative to the surface of the support, and it is assumed that the chromophore is separated from the support by lipid layers. Data for vertical (solid line), parallel (dashed line), and space-averaged $\left(48^{\circ}\right)$ (dotted line) orientation are shown together with experimental points (squares).

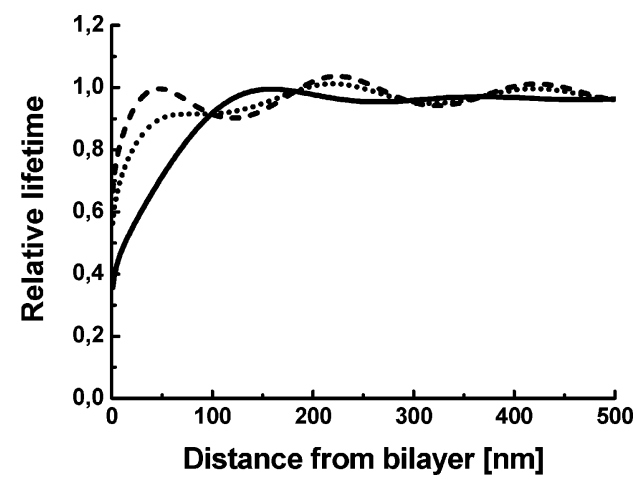

Figure 6. Calculated dependences of relative lifetime of free dye (assuming quantum yield $=1$ ) in buffer on its distance from SPBs created on a $173-\mathrm{nm}$ thick ITO layer on glass surface. The shown distance is measured relative to the surface of SPBs, and it is assumed that the chromophore is separated from the SPBs by water. This leads to larger values of relative lifetimes at zero distance than in Figure 4. Data for vertical (solid line), parallel (dashed line), and space-averaged $\left(54^{\circ}\right)$ (dotted line) orientation are shown.

broader use of FLCS experiments. SPBs directly adsorbed onto oxidized silicon can serve as an example. While in the pure SPBs system the lifetime is too short as well as the fluorescence intensity is too low for FLCS experiments, use of seven layers of DPPA decreases the quenching efficiency and negative interference of both the excitation and emission light and provides a reasonable fluorescence intensity with a lifetime being almost one-half the value of the unquenched dye in SUVs. Figures 4-6 demonstrate that for the averaged orientation the lifetime almost reaches its bulk values for distances shorter than $50 \mathrm{~nm}$. Compared with the standard $z$-size of the focal volume $(1-3 \mu \mathrm{m})$, this means that for the FLCS experiments on samples containing free diffusing vesicles only a very small fraction of labeled vesicles located very close to the surface exhibits a significantly shortened lifetime and, consequently, interferes with the signal from the membrane.

The glass support covered with a conductive 173-nm thick ITO layer appears to be most suitable for FLCS experiments aiming at simultaneous characterization of lateral diffusion of labeled species within SPBs and of three-dimensional diffusion of the same species in solution above the SPBs. Though the resonance energy transfer to the conducting ITO layer shortens the lifetime by a factor of 3.1, the remaining fluorescence intensity is still high enough for FLCS experiments. Oxidized silicon is not as suitable as ITO for two reasons. First, quenching persists

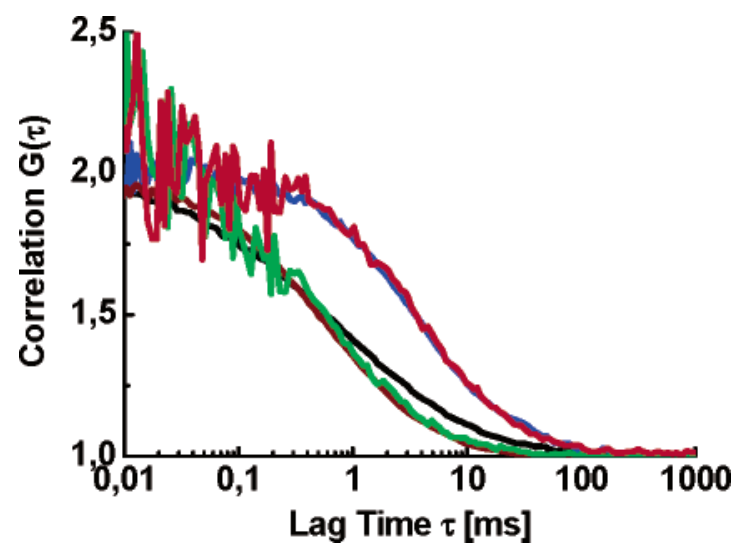

Figure 7. Normalized autocorrelation curves for (a) a mixture of SPBs with SUVs $((\tau(\mathrm{F})=1.8$ and $5.6 \mathrm{~ns}$, respectively) (black line), (b) SPBs $(\tau(\mathrm{F})=1.8 \mathrm{~ns})$ in pure buffer (blue line), (c) SUVs $(\tau(\mathrm{F})$ $=5.6 \mathrm{~ns})$ in solution (brown line), $(\mathrm{d}) \mathrm{SPBs}(\tau(\mathrm{F})=1.8 \mathrm{~ns})$ calculated from the mixture of SPBs and SUVs using FLCS (red line), and (e) SUVs $(\tau(\mathrm{F})=5.6 \mathrm{~ns})$ calculated from the mixture of SPBs and SUVs using FLCS (green line). The SPBs and SUVs consisted of a DOPC/DOPS mixture 4/1 labeled 1:100 000 with $\beta$-C8-BODIPY 500/510 C5-HPC. SPBs were created on glass covered with $173 \mathrm{~nm}$ ITO.

to larger dye-support distances than in the case of ITO. The second reason is connected with the brightness of dyes in SPBs. Owing to its high refractive index, silicon exhibits a negative interference for excitation and emission light at short distances from the support. ${ }^{23,24}$ This reduces the brightness of the dyes in a single bilayer to such an extent that meaningful FLCS measurements are almost impossible. On the other hand, ITO exhibits a rather low refractive index (2.043); the interference is much lower and, consequently, provides for sufficiently high brightness of dyes in SPBs.

FLCS Experiments for Simultaneous Characterization of Two- and Three-Dimensional Diffusion. An interesting feature of light-absorbing surfaces was shown in the preceding paragraphs. Due to their optical properties they substantially quench fluorescence of dyes situated close to them, but the quenching disappears within tens of nanometers toward the solution. This feature can be used for differentiating between 2D and 3D motion of the same labeled species by means of FLCS.

The idea underlying the performed experiments is illustrated in Figure 1. After exposure of the hydrophilic surface to a suspension of SUVs, confluent SPBs were formed. The redundant SUVs remained in solution. Due to the effect of the solid support the lifetime of the dye in SPBs is considerably shorter than the lifetime in SUVs. The laser beam is focused at the interface. (We use an inverted microscope; the beam is coming from the bottom through the water solution.) It excites the dye molecules in both SPBs and SUVs. This results in a very complicated autocorrelation curve containing a term for 2D diffusion (SPBs) and a term for confined 3D diffusion (SUVs). The approach presented in this paper allows separating of the complicated autocorrelation function into the corresponding autocorrelation functions for $2 \mathrm{D}$ diffusion within the planar bilayers as well as for 3D diffusion of the SUVs in solution. Moreover, a possible interaction between SUVs and SPBs can be addressed by calculating the crosscorrelation functions.

FLCS experiments were performed on SPBs directly adsorbed onto ITO-covered glass $(\tau(\mathrm{F})=1.8 \mathrm{~ns})$ (Figure 7$)$ as well as on

(23) Lambacher, A.; Fromherz, P. J. Opt. Soc. Am. B: Opt. Phys. 2002, 19 (6), 1435-1453. 216. 


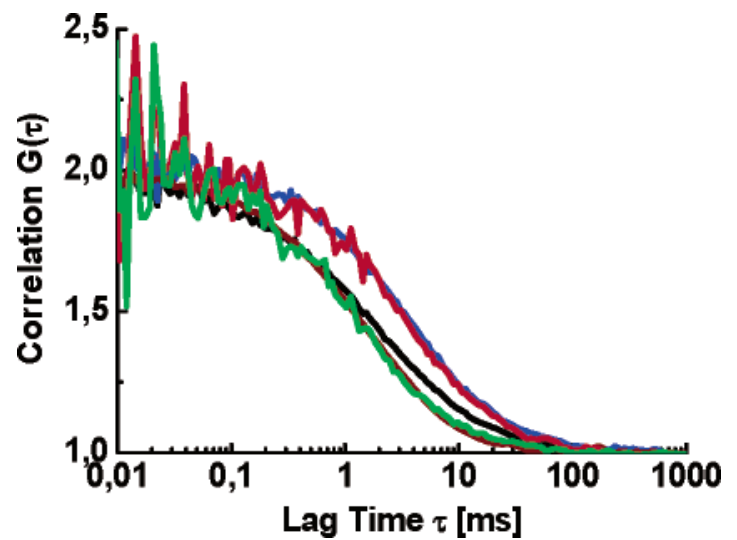

Figure 8. Normalized autocorrelation curves for (a) a mixture of lipid monolayer with SUVs $((\tau(\mathrm{F})=3.0$ and $5.6 \mathrm{~ns}$, respectively) (black line), (b) lipid monolayer $(\tau(\mathrm{F})=3.0 \mathrm{~ns}$ ) in pure buffer (blue line), (c) SUVs $(\tau(\mathrm{F})=5.6 \mathrm{~ns})$ in solution (brown line), (d) lipid monolayer $(\tau(\mathrm{F})=3.0 \mathrm{~ns})$ calculated from the mixture of lipid monolayer and SUVs using FLCS (red line), and (e) SUVs $(\tau(\mathrm{F})=$ $5.6 \mathrm{~ns}$ ) calculated from the mixture of SPBs and SUVs using FLCS (green line). The lipid monolayer and SUVs consisted of a DOPC/ DOPS mixture 4/1 labeled 1:100 000 with $\beta$-C8-BODIPY 500/510 C5-HPC. The lipid monolayer was created on seven monolayers of DPPA deposited on naturally oxidized silicon.

a DOPC/DOPS monolayer adsorbed onto seven DPPA layers on oxidized silicon $(\tau(\mathrm{F})=3.0 \mathrm{~ns}$ ) (Figure 8 ). Figures 7 and 8 show the normalized correlation curves of the entire system and the resulting autocorrelation curves calculated for lateral diffusion as well as for the confined three-dimensional SUVs diffusion. No cross-correlation was observed (curve not shown) since there was no exchange of lipid molecules between SPBs and the vesicles under the conditions employed. The recorded autocorrelation curves for labeled SPBs after flushing away the vesicles (a purely 2D diffusion) and the autocorrelation curves for labeled vesicles in solution using standard FCS are shown for comparison.

The presented concept can be proved by comparing the autocorrelation functions obtained for either SPBs or monolayers from the mixed SPB/vesicle systems with those obtained from the isolated planar lipid layers or for SUVs again obtained for the mixed SPB/vesicle systems with those obtained for pure SUVs (see Figures 7 and 8). The visual comparison is supported by the fact that the diffusion coefficients obtained by FLCS for both investigated planar bilayer systems are identical with those obtained for the vesicle-free systems. $\left(D=4.2 \pm 0.4 \times 10^{-12}\right.$ and $4.0 \pm 0.4 \times 10^{-12} \mathrm{~m}^{2} \mathrm{~s}^{-1}$ for DOPC/DOPS 4/1 SPBs and DOPC/DOPS $4 / 1$ monolayers on DPPA, respectively). The obtained values are in agreement with those reported in a previous paper using the " $z$-scan" approach. ${ }^{4}$ Please note that the autocorrelation functions for pure SUVs cannot be fitted to a simple one- or two-component diffusion model, which may have two reasons: (1) SUVs are too large for the pointlike molecule assumption underlying the use of these models ${ }^{1}$ and (2) 2D diffusion of the fluorescent label within the vesicle's bilayer happens on a comparable time scale as the 3D diffusion of the whole vesicle, thus making the autocorrelation function more complex. This subject is presently under investigation in our laboratory.

\section{Perspectives}

To fully exploit FLCS in supported phospholipid bilayers' research, the lifetimes of the membrane-associated chromophores have to be tuned. Since the stacking technique for defined multilayer spacers is quite laborious and requires a LB trough, variation of the supports or controlled chemical modification of a given support appear to be more practicable ways to control the lifetimes.

Since the dependence of lifetimes on the dye-support distance for light-absorbing surfaces is rather steep (Figures 4 and 5), use of headgroup-labeled lipids leads already to a significant difference in lifetime between chromophores located in the inner and outer layer of SPBs. This may allow one to characterize diffusion in both layers of SPBs simultaneously and independently using FLCS. This idea is currently under investigation.

Lateral diffusion of weakly bound proteins, e.g., like the blood coagulation protein prothrombin, is very difficult to characterize. ${ }^{25}$ Since free and bound proteins are in equilibrium, flushing away free protein results in protein desorption. Thus, it is not possible to define conditions for a system containing membrane-bound proteins but no free protein. Under any experimental conditions the fluorescence signal from the free protein thus interferes with the signal that originates in the bound protein. However, the lifetime of the dye attached to the membrane-bound protein might be controlled using the approach demonstrated in this study. By analogy with the aforementioned vesicle experiments, FLCS might then enable one to characterize simultaneously twodimensional protein diffusion on SPBs and three-dimensional protein diffusion in bulk. Moreover, the cross-correlation analysis might provide direct information on protein binding rates.

Acknowledgment. We thank the Grant Agency of the Czech Republic (A.B. and V.F. via 203/05/2308) and the Ministry of Education, Youth and Sports of the Czech Republic (M.H. via LC06063 and A.D. via Institutional Research Plan No. AV0Z10100522) for financial support.

\section{LA061573D}

(25) Huang, Z. P.; Pearce, K. H.; Thompson, N. L. Biophys. J. 1994, 67 (4), $1754-1766$. 